\title{
Engineered Cardiac Tissues Generated from Immature Cardiac and Stem Cell-Derived Cells: Multiple Approaches and Outcomes
}

\author{
Bradley B. Keller, Fei Ye, Fangping Yuan, Hiren Trada, \\ Joseph P. Tinney, Kevin M. Walsh, and Hidetoshi Masumoto
}

\begin{abstract}
The translation of in vitro engineered cardiac tissues (ECTs) from immature cardiac and stem cell-derived cells toward clinical therapies is benefiting from the following major advances: (1) rapid progress in the generation of immature cardiac cells from the cardiac and noncardiac cells of multiple species including normal and disease human cells, (2) incorporation of multiple cell lineages into 3D tissues, (3) multiple scalable 3D formulations including injectable gels and implantable tissues, and (4) insights into the regulation of cardiomyocyte proliferation and functional maturation. These advances are based on insights gained from investigating the regulation of cardiac morphogenesis and adaptation. Our lab continues to explore this approach, including changes in gene expression that occur in response to mechanical loading and tyrosine kinase inhibition, the incorporation of vascular fragments into ECTs, and the fabrication of porous implantable electrical sensors for in vitro conditioning and postimplantation testing. Significant challenges remain including optimizing ECT survival postimplantation and limited evidence of ECT functional coupling to the recipient myocardium. One clear focus of current research is the optimization and expansion of the cellular constituents, including CM, required for clinical-grade ECTs. Another major area of investigation will be large animal preclinical models that more accurately represent human $\mathrm{CV}$ failure and that can generate data in support of regulatory approval for phase I human clinical trials. The generation of reproducible human ECTs creates the opportunity to develop in vitro myocardial surrogate tissues for novel drug therapeutics and toxicity assays.
\end{abstract}

B.B. Keller $(\bowtie) \cdot$ F. Ye • F. Yuan • H. Trada • J.P. Tinney • K.M. Walsh • H. Masumoto Kosair Charities Pediatric Heart Research Program, Cardiovascular Innovation Institute, University of Louisville, 302 E. Muhammad Ali Blvd, Louisville, KY 40202, USA e-mail: brad.keller@louisville.edu

T. Nakanishi et al. (eds.), Etiology and Morphogenesis of Congenital Heart Disease, DOI 10.1007/978-4-431-54628-3_46 


\section{Keywords}

Cardiomyocytes $\bullet$ Cardiac repair and regeneration • Engineered cardiac tissues • Stem cells

\subsection{Introduction}

Following significant cardiac injury, the postnatal human heart lacks the ability to restore lost myocardium, resulting in an adaptive response that often ultimately leads to progressive cardiac dysfunction, morbidity, and mortality. There are currently many strategies for cardiac "cellular therapy" undergoing both preclinical and clinical trials [1-4]. While there has been modest success with improvement in cardiac function in some of the early human clinical trials, it is clear that injected or implanted cells do not survive, and functional improvement occurs via paracrine mechanisms. In contrast, rapid advances in tissue engineering over the past two decades have resulted in the generation of functional, multicellular, 3D cardiac tissues with the potential for translation to human cardiac repair and regeneration [5-8]. This chapter provides a concise overview of some of the key issues in the generation, maturation, and translation of these engineered cardiac tissues (ECTs).

\subsection{A Broad View of Bioengineering Cardiac Tissues}

The bioengineering process for complex tissues begins with an understanding of the cellular and noncellular constituents of the target tissue [9]. For replacement myocardium, the major cellular constituents include cardiomyocytes (CM), fibroblasts, and vessel-associated cells. There are also numerous extracellular matrix (ECM) constituents including collagen, fibronectin, laminin, and multiple growth factors bound within the ECM. Of course, the neonatal myocardium and the adult myocardium have vastly different profiles for cellular and noncellular constituents, architecture, and biomechanical properties. While all currently successful ECTs are constructed using immature cells and simplified ECM components, the target tissue is usually mature myocardium. The success of ECT survival, integration, and functional maturation depends on the ability of these ECT constituents to acquire "mature" fates.

\subsection{Immature Cells for Engineered Cardiac Tissues}

Because the goal for cardiac regeneration is the restoration of functionally coupled, working myocardium, a variety of cell sources with the potential to generate CM are under investigation. Immature $\mathrm{CM}$ can be isolated from the hearts of developing chick, mouse, and rat embryos to generate ECTs for preclinical investigation (Fig. 46.1) [5-7]. These cells mature in vivo or in vitro along timelines proportional 


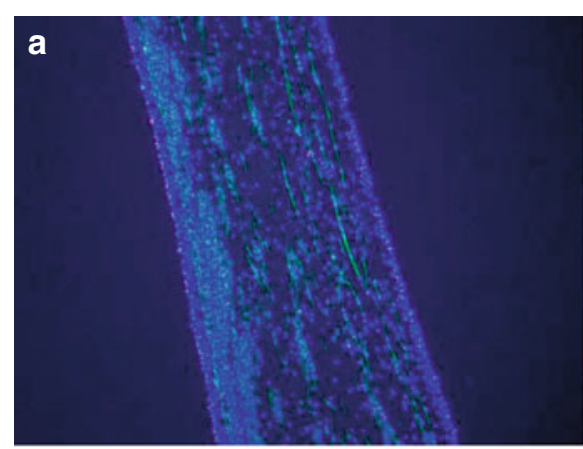

C

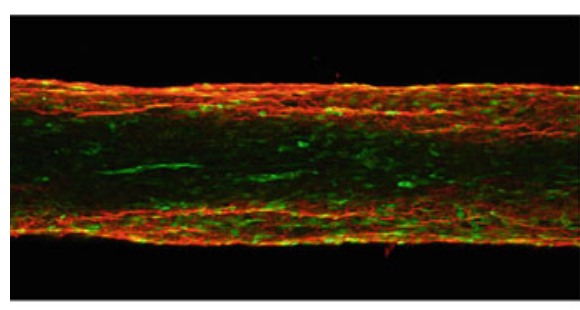

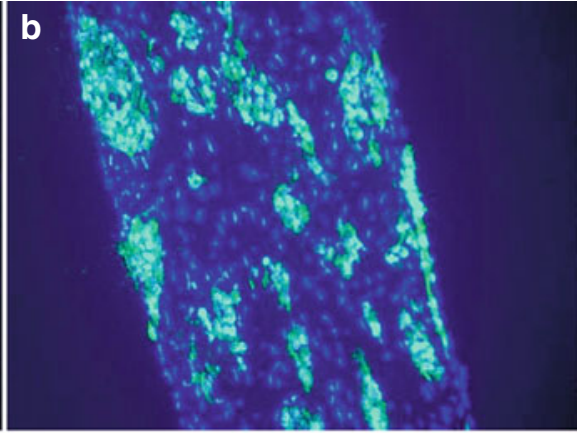

d

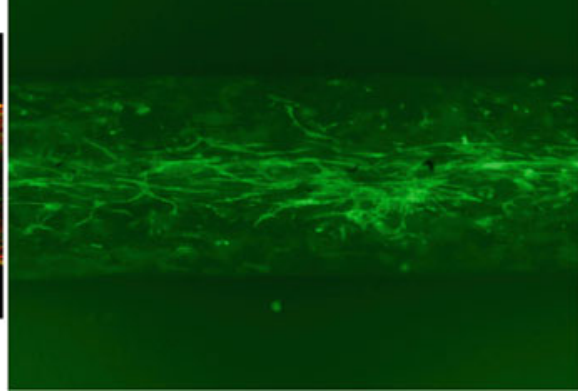

Fig. 46.1 Representative engineered cardiac tissues (ECTs) derived from (a) embryonic chick heart cells; (b) human-iPS-derived cardiomyocytes; (c) embryonic rat heart cells and rat adipose vascular fragments; and (d) enlarged image of vascular fragments within a rat ECT. Staining for (a, b) are blue (DAPI, nuclei), green (cardiac troponin T), and red (EdU). Staining for (c, d) are red (alpha actinin) and green (GFP+vascular fragments). Images (a, c) are $20 \times$ magnification; images (b, d) are $40 \times$ magnification (Keller lab, unpublished)

to the gestational length of their species of origin. A variety of stem cell sources (embryonic stem cells, induced pluripotent stem cells, cardiac stem cells, adipose stem cells, etc.) have also been used to generate immature CM using a variety of CM lineage specification and selection protocols [10-14]. Not surprisingly, stem cell populations can be rapidly expanded in vitro along with the induction of cardiac lineages; however, their functional maturation remains a major technical challenge [15-17]. Because human cells are required for clinical translation, the optimization of protocols that can generate large quantities of functional human CM is a high priority for cardiac repair strategies. Further, there may be advantages to generating ECTs that contain both cardiac and vascular lineage cells to accelerate angiogenesis and vascular perfusion of implanted ECTs [11-13]. 


\subsection{Various Formulations for Engineered Cardiac Tissues}

The constructs used for cardiac tissue repair include the implantation of multicellular cardiospheres [18], various formulations of 2D cellular sheets [19-22], and various formulations of 3D tissues [5-8, 13]. The composition of the noncellular constituents varies from minimal constituents for cardiosphere clusters to a range of ECM components [23, 24] and growth factors [25-27] selected for their ability to facilitate CM survival and functional maturation. Some of the ECT formulations allow for in vitro preconditioning strategies that can stimulate cell proliferation and/or maturation $[5,6]$. While there can be wide variation in the formulation of ECTs used for preclinical studies, all constituents used to generate ECTs for human use are required to conform to strict FDA regulatory guidelines that include the elimination of all sources for potential infectious agents and/or toxins and the generation of clinical-use materials using good manufacturing practices [28].

\subsection{In Vitro ECT Findings}

Immature CM survive, proliferate, and functionally mature rapidly within ECTs as quantified by standard measures of cell number, gene expression profiling (Fig. 46.2) [29], sarcomeric protein content, electrophysiologic properties, and the ability to generate substantial force $[5-7,13]$.

Maturing functional syncytia can include CM, myofibroblasts, and vascular cells with a functional advantage noted for multiple lineage constructs over pure CM-derived ECTs [11-13]. Conditioning protocols with the intent of accelerating

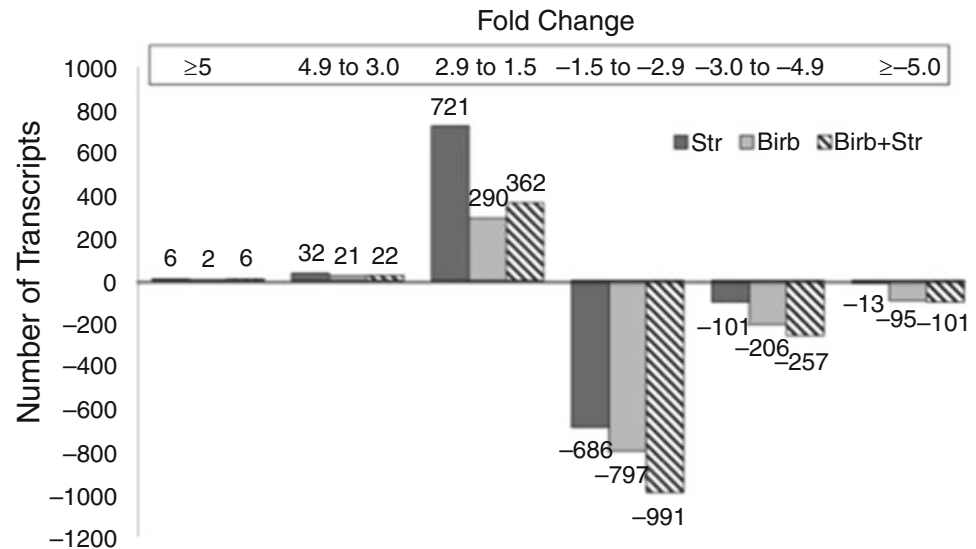

Fig. 46.2 Changes in rat ECT gene expression in response to mechanical loading and/or p38MAPK inhibition. ECT transcript expression changes at least 1.5 -fold measured by microarray in response to stretch (dark solid bar), the p38MAPK inhibitor BIRB796 (gray solid bar), or stretch+BIRB796 (dashed bar). Note that most transcripts increased by less than threefold (above the X-axis) or decreased by less than fivefold (below the X-axis) [29] 

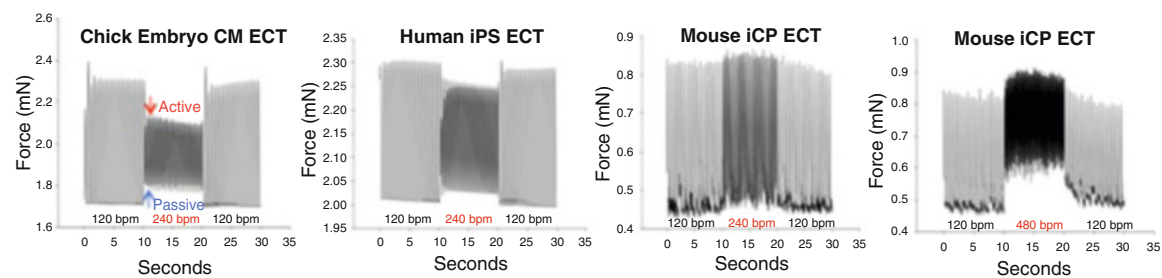

Fig. 46.3 Representative force-frequency relations for chick embryo-derived ECT and humaniPS-CM-derived ECT. Force-frequency relations quantify force generation in response to increased beat rate and reflect ECT maturational ability to release and restore $\mathrm{Ca}^{2+}$. Chick embryo and h-iPS ECT showed increased passive force and reduced active force as beat rate increased from 120 to $240 \mathrm{bpm}$ consistent with immature $\mathrm{Ca}^{2+}$ release during contraction and limited $\mathrm{Ca}^{2+}$ sequestration at faster rates (Keller lab, unpublished)

cell proliferation and/or maturation include the use of exogenous paracrine factors $[15,26]$, electrical stimulation [17, 27], and/or mechanical conditioning $[6,13]$. Structural CM maturation is readily documented using immunohistochemical stains for sarcomeric proteins, gap junctions, and the presence of ion channels [30]. Functional CM maturation is documented by decreasing pacing voltage thresholds to initiate pacing, intrinsic rates and maximal beat rates in response to electrical stimulation, increasing rates of electrical conduction across ECTs, developed force in response to pacing, and both force-length and force-frequency relations reflecting increased contractility and increased calcium cycling efficiency (Fig. 46.3). ECTs generated from human-derived cells are now proposed as in vitro models for human diseases as well as surrogate models to detect drug toxicity prior to clinical trials $[31,32]$.

\subsection{In Vivo ECT Findings}

Ultimately, ECTs require in vivo implantation to assess survival, structural integration to the recipient myocardium, functional integration, and evidence for recovery of lost cardiac function. Preclinical implantation models have been primarily small animals (rodents); however, there is an increasing experience with the preclinical testing of ECTs using large animal models including pigs [22] and, eventually, nonhuman primates. The in vivo results have been very encouraging and confirm the capacity of implanted ECTs to survive, functionally couple, and recover damaged myocardium within the context of the experimental design [33]. There are several challenges to the interpretation of in vivo ECT studies. First, many of the studies involve the implantation of ECTs into immune-compromised animals. These studies are required to validate the capacity of implanted ECTs to functionally couple to recipient myocardium but underestimate the rapid inflammatory degradation that occurs as evidenced by the presence of macrophage-associated arginase and reduced ECT post-implant cellularity, even in syngeneic animals (Fig. 46.4) [13, 30]. 

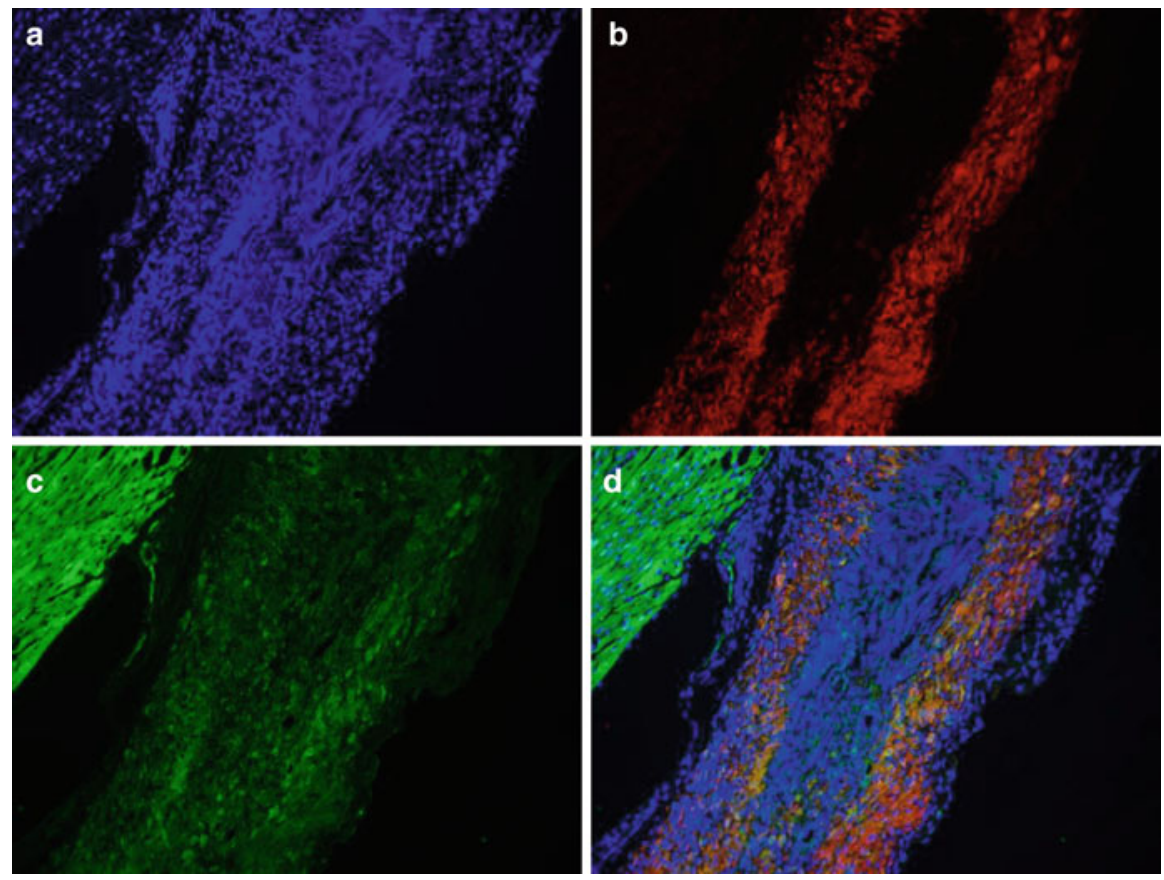

Fig. 46.4 Representative postimplantation histology for rat embryo-derived ECT implanted onto the epicardial surface of a syngeneic adult male rat 2 weeks after coronary artery ligation-induced myocardial infarction. (a) Blue (DAPI, nuclei), (b) red (arginase marker for macrophages), (c) green (GFP+ for recipient myocardium and inflammatory cells), (d) merged image. Images are $10 \times$ magnification. Note the high cellularity of the recipient myocardium (upper left corner) and the implanted ECT. Note the increased presence of arginase along the outer margins of the implanted ECT, consistent with the higher cell density noted in ECT (Keller lab, unpublished)

Second, the in vivo results are often reported after relatively short periods that may not reflect long-term, sustained functional recovery [13]. Finally, the acute surgical models of cardiac injury in preclinical models often do not fully represent the human disease state with medical comorbidities and recurrent episodes of ischemia/injury. To date there are too few studies in large animal models to validate the feasibility of scaling up CM and ECT production for cardiac repair and to compare direct cell delivery strategies to ECT implantation strategies for short- and longer-term efficacy.

\subsection{Future Directions}

One clear focus of current and future research is the optimization and expansion of the cellular constituents, including CM, required for clinical-grade ECTs. Ultimately, these cells will need to be from human sources and have minimal immunogenic profiles. Another major area of investigation will be large animal preclinical 
models that more accurately represent human CV failure and that can generate data in support of regulatory approval for phase I human clinical trials. Related to these large animal models will be innovations in the ability to manufacture large-scale ECTs and to implant them with minimally invasive techniques. Although it is beyond the scope of this chapter, the generation of reproducible human ECTs creates the opportunity to develop in vitro myocardial surrogate tissues for novel drug therapeutics and toxicity assays.

Open Access This chapter is distributed under the terms of the Creative Commons AttributionNoncommercial 2.5 License (http://creativecommons.org/licenses/by-nc/2.5/) which permits any noncommercial use, distribution, and reproduction in any medium, provided the original author(s) and source are credited.

The images or other third party material in this chapter are included in the work's Creative Commons license, unless indicated otherwise in the credit line; if such material is not included in the work's Creative Commons license and the respective action is not permitted by statutory regulation, users will need to obtain permission from the license holder to duplicate, adapt or reproduce the material.

\section{References}

1. Clifford DM, Fisher SA, Brunskill SJ, et al. Stem cell treatment for acute myocardial infarction. Cochrane Database Syst Rev. 2012;2:CD006536.

2. Kandala J, Upadhyay GA, Pokushalov E, et al. Meta-analysis of stem cell therapy in chronic ischemic cardiomyopathy. Am J Cardiol. 2013;112:217-25.

3. Mielewczik M, Cole GD, Nowbar AN, et al. The C-CURE randomized clinical trial (Cardiopoietic stem Cell therapy in heart failURE). J Am Coll Cardiol. 2013;62(25):2453.

4. Heldman AW, DiFede DL, Fishman JE, et al. Transendocardial mesenchymal stem cells and mononuclear bone marrow cells for ischemic cardiomyopathy: the TAC-HFT randomized trial. JAMA. 2014;311(1):62-73.

5. Zimmermann WH, Melnychenko I, Wasmeier G, et al. Engineered heart tissue grafts improve systolic and diastolic function in infarcted rat hearts. Nat Med. 2006;12(4):452-8.

6. Tobita K, Liu LJ, Janczewski AM, et al. Engineered early embryonic cardiac tissue retains proliferative and contractile properties of developing embryonic myocardium. Am J Physiol Heart Circ Physiol. 2006;291(4):H1829-37.

7. de Lange WJ, Hegge LF, Grimes AC, et al. Neonatal mouse-derived engineered cardiac tissue: a novel model system for studying genetic heart disease. Circ Res. 2011;109(1):8-19.

8. Turnbull IC, Karakikes I, Serrao GW, et al. Advancing functional engineered cardiac tissues toward a preclinical model of human myocardium. FASEB J. 2014;28(2):644-54.

9. Mikos AG, Herring SW, Ochareon P, et al. Engineering complex tissues. Tissue Eng. 2006;12 (12):3307-39.

10. Duan Y, Kettenhofen R, Jovinge S, et al. Engraftment of engineered ES cell-derived cardiomyocytes but not $\mathrm{BM}$ cells restores contractile function to the infarcted myocardium. J Exp Med. 2006;203(10):2315-27.

11. Stevens KR, Kreutziger KL, Dupras, et al. Physiological function and transplantation of scaffold-free and vascularized human cardiac muscle tissue. PNAS. 2009;106(39):16568-73.

12. Lesman A, Habib M, Caspi $O$, et al. Transplantation of a tissue-engineered human vascularized cardiac muscle. Tissue Eng Part A. 2010;16(1):115-25.

13. Tulloch NL, Muskheli V, Razumova MV, et al. Growth of engineered human myocardium with mechanical loading and vascular coculture. Circ Res. 2011;109(1):47-59. 
14. Zhang D, Shadrin IY, Lam J, et al. Tissue-engineered cardiac patch for advanced functional maturation of human ESC-derived cardiomyocytes. Biomaterials. 2013;34(23):5813-20.

15. Pillekamp F, Haustein M, Khalil M, et al. Contractile properties of early human embryonic stem cell-derived cardiomyocytes: beta-adrenergic stimulation induces positive chronotropy and lusitropy but not inotropy. Stem Cells Dev. 2012;21(12):2111-21.

16. Yang X, Pabon L, Murry CE. Engineering adolescence: maturation of human pluripotent stem cell-derived cardiomyocytes. Circ Res. 2014;114(3):511-23.

17. Nunes SS, Miklas JW, Liu J, et al. Biowire: a platform for maturation of human pluripotent stem cell-derived cardiomyocytes. Nat Methods. 2013;10(8):781-7.

18. Malliaras K, Makkar RR, Smith RR, et al. Intracoronary cardiosphere-derived cells after myocardial infarction: evidence of therapeutic regeneration in the final 1-year results of the CADUCEUS trial (CArdiosphere-Derived aUtologous stem CElls to reverse ventricUlar dySfunction). J Am Coll Cardiol. 2014;63(2):110-22.

19. Shimizu T, Yamato M, Kikuchi A, Okano T. Cell sheet engineering for myocardial tissue reconstruction. Biomaterials. 2003;24(13):2309-16.

20. Fujita J, Itabashi Y, Seki T, et al. Myocardial cell sheet therapy and cardiac function. Am J Physiol Heart Circ Physiol. 2012;303(10):H1169-82.

21. Matsuura K, Haraguchi Y, Shimizu T, Okano T. Cell sheet transplantation for heart tissue repair. J Control Release. 2013;169(3):336-40.

22. Kawamura M, Miyagawa S, Fukushima S, et al. Enhanced survival of transplanted human induced pluripotent stem cell-derived cardiomyocytes by the combination of cell sheets with the pedicled omental flap technique in a porcine heart. Circulation. 2013;128(11 Suppl 1): S87-94.

23. Black LD, Meyers JD, Weinbaum JS, et al. Cell-induced alignment augments twitch force in fibrin gel-based engineered myocardium via gap junction modification. Tissue Eng Part A. 2009;15(10):3099-108.

24. Yuan Ye K, Sullivan KE, Black LD. Encapsulation of cardiomyocytes in a fibrin hydrogel for cardiac tissue engineering. J Vis Exp. 2011;55:pii:3251.

25. Chiu LL, Radisic M, Vunjak-Novakovic G. Bioactive scaffolds for engineering vascularized cardiac tissues. Macromol Biosci. 2010;10(11):1286-301.

26. Bearzi C, Gargioli C, Baci D, et al. PIGF-MMP9-engineered iPS cells supported on a PEG-fibrinogen hydrogel scaffold possess an enhanced capacity to repair damaged myocardium. Cell Death Dis. 2014;5:e1053.

27. Park H, Larson BL, Kolewe ME, et al. Biomimetic scaffold combined with electrical stimulation and growth factor promotes tissue engineered cardiac development. Exp Cell Res. 2014;321(2):297-306.

28. 7341.002 - inspection of human cells, tissues, and cellular and tissue-based products (HCT/Ps). US Food and Drug Administration. http://www.fda.gov/biologicsbloodvaccines/ guidancecomplianceregulatoryinformation/complianceactivities/enforcement/ complianceprograms/ucm095207.htm

29. Ye F, Yuan F, Li X, et al. Gene expression profiles in engineered cardiac tissues respond to mechanical loading and inhibition of tyrosine kinases. Physiol Rep. 2013;1(5):e00078.

30. Tiburcy M, Didié M, Boy O, et al. Terminal differentiation, advanced organotypic maturation, and modeling of hypertrophic growth in engineered heart tissue. Circ Res. 2011;109 (10):1105-14.

31. Lalit PA, Hei DJ, Raval AN, et al. Induced pluripotent stem cells for post-myocardial infarction repair: remarkable opportunities and challenges. Circ Res. 2014;114(8):1328-45.

32. Vunjak Novakovic G, Eschenhagen T, et al. Myocardial tissue engineering: in vitro models. Cold Spring Harb Perspect Med. 2014;4(3): pii: a014076.

33. Fujimoto KL, Clause KC, Liu LJ, et al. Engineered fetal cardiac graft preserves its cardiomyocyte proliferation within postinfarcted myocardium and sustains cardiac function. Tissue Eng Part A. 2011;17(5-6):585-96. 